\title{
Declining Participation in Research Studies
}

\author{
Cynthia L. Arfken Richard Balon \\ Department of Psychiatry and Behavioral Neurosciences, Wayne State University School of Medicine, \\ Detroit, Mich., USA
}

\section{Introduction}

Participation rates in research studies, whether measured as the percentage of people eligible from a defined population who are recruited or the percentage of recruited people who participate in prespecified follow-ups, are dropping in the USA [1] and perhaps are or will be elsewhere. With that drop, confidence in research findings is dropping, too. This phenomenon is not limited to observational studies/surveys; it is also occurring in experimental studies and clinical trials. The ideal is, of course, to have a $100 \%$ participation rate. With a perfect participation rate at recruitment, we are confident that the results (assuming they have internal validity) are generalizable. But the perfect participation rate is rarely possible for a reasonable sample size of a public health or clinically important target population. At what point do we as readers/reviewers, researchers and policymakers become concerned with less than perfect participation? Does it matter if it is an online survey or mail/telephone/in person interview? What can be done to improve the participation rate? The answers to these questions are critical to the research enterprise and the public's acceptance of our findings. We discuss each of these questions below.

\section{At What Point Do We as Readers/Reviewers, Researchers and Policymakers Become Concerned?}

A quick review of some standard research texts did not reveal a number that participation rate had to exceed to be scientifically acceptable. Nonetheless, in an informal poll, researchers quickly mentioned 'above $90 \%$,' ' $80 \%$ ' or ' $60 \%$ ' as the target participation rate for recruitment. From this admittedly convenience sample, it appears that informal standards are being used. On the one hand, a selected number has the same drawback as using $\mathrm{p}=0.05$ to determine statistical significance. With $80 \%$ as the arbitrary target, $80.1 \%$ would be deemed scientifically acceptable and $79.9 \%$ would be rejected. Picking such a magical number would mean that people would invent, and have invented, ways to inflate their participation rate to reach an 'acceptable' rate. Due to this creativity, it is critical that the calculation of the participation rates for recruitment and for follow-up, when applicable, are reported. This transparency is reflected in the CONSORT requirement of presenting the flow of participants throughout a clinical trial.

The second drawback to identifying a participation rate as acceptable is more insidious. As anything short of $100 \%$ participation in any kind of study has the potential of bias, it is vital to the interpretation of findings that the pattern of nonparticipation, like the pattern of missing values when selecting analytical strategies, be examined for bias. The US census has a high participation using a mixed mode of data collection (postal survey followed by in person interviews) but systematically misses disadvantaged people. In contrast, the ongoing telephone-administered US Behavioral Risk Factor Survey has a participation rate below $60 \%$ but no evidence of bias.

Examining for bias can involve multiple steps. For studies with follow-up, the analysis includes contrasting who was lost to follow-up (and why) to those for whom the researcher has data. For recruitment, the approach is more difficult. Galea and Tracy [1] highlight increased

\section{KARGER \\ Fax +41 613061234 E-Mail karger@karger.ch} www.karger.com

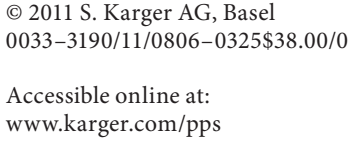

Cynthia L. Arfken, PhD

Wayne State University

2761 E. Jefferson

Detroit, MI 48207 (USA)

Tel. +1 313993 3490, E-Mail carfken@med.wayne.edu 
difficulty in obtaining this information in the USA due to regulations, such as the Health Insurance Portability and Accountability Act and other confidentiality policies or measures. However, if characteristics of the target population are known, the researcher can compare them with the characteristics of the recruited sample. The researcher can also incorporate a substudy with minimal data collection within the larger study to characterize people who did not agree to the full study. Qualitative interviews with the target population may suggest reasons why some people refused to participate in a specific study. Presenting vignettes of potential study designs and components has been used [2]. A walkthrough of the recruitment procedure by an investigator may also reveal barriers resulting in systematic bias. For sampling based upon where the person lives, geographic distribution of responders and nonresponders can be compared [3]. Recruiting either professional volunteers (i.e. people who participate in multiple studies for the compensation) or symptomatic volunteers [4] can introduce bias.

From past studies, we can generalize that people are more likely to participate if the researcher has built trust with the target population and there is a personal appeal to participate, the benefit can be easily seen and valued by the potential participant, the procedures are not extensive/invasive/burdensome, and the people approached have a favorable view/experience with research. However, these are generalizations and may change over time.

Galea and Tracy [1] argued that participation rates are dropping due to the sheer number of studies being performed, especially those over the Internet. Thus, the movement to have solid numbers for policy decisions, train clinicians to be research literate, communicate with college students by administration and, at least in the USA, marketing disguised as survey research means people are feeling overwhelmed and less trustful of 'research'. They also argued that willingness to spend time in other community activities ('volunteerism') is also declining as well as overall free time in the USA and other western countries [1]. It is doubtful that either of those conditions will change in the near future.

Another barrier or deterrent to participation is the time commitment and the formalized consent process required in the USA. At least one study we know had a longer consent process than the intervention being studied. Informed consent forms in cancer research studies routinely reach double-digit numbers of pages. One of us recently reviewed a protocol with an informed consent form that was 34 pages long. We have also personally heard potential participants say they do not trust consent sheets because, in the words of the people approached, they are written by lawyers to protect the university. However, even between universities there are different requirements. In an online survey one of us is currently doing, our information sheet requires scrolling down multiple screens to reach the agree button. In contrast, at another university implementing the same survey, the information sheet is condensed to one screen. This is not to say that consent is bad; it is to say that we need to look at this from the research participant's perspective.

The direction of the biases may be suggested by conducting the steps mentioned above, but there will always remain some nondirectional bias. We advocate along with others that as a general rule avoid highlighting small effects that could be easily due to confounding. Formal sensitivity analysis can also quantify the extent of the bias necessary to eliminate the finding [5]. Small effects consistent with the theoretical framework may still be published but need to be replicated by different research groups before general acceptance of them.

\section{Does It Matter if It Is an Online Survey or a Mail/Telephone/in Person Interview?}

The participation rate is important across all methods of data collection. The same high standard in participation whether someone believes it is $90 \%$ or $60 \%$ is needed for each method of data collection as the potential for bias can be present in all these methods. Obviously, there can be differences in the quality of the data collected by mode of data collection, especially in the ability to clarify or follow up on questions and recognize fatigue or deception in the participant. For online surveys, researchers have recognized the challenges and developed guidelines for them [6]. Pointing to the need of more research on the technique itself is the fact that there is a journal devoted to advancing the science of online surveys, the Journal of Medical Internet Research. For this commentary, we will only emphasize that online and postal surveys have to be especially diligent to be clear and short.

Due to its ease of constructing and low cost of administering - no postage, no research assistants contacting people - online surveys are particularly appealing to researchers with low budgets and, from the number sent to us, are multiplying. Online surveys must still address questions of who is the target population and what is the 
sample size needed. If mass e-mailing is used (e.g. to all medical students in the USA), the researcher still needs to stop and think about who and how many people are realistically going to respond. Concentrating on fewer schools geographically dispersed and achieving a high participation rate would be preferable to a low participation rate nationally.

We recently received an online survey (funded by a reputable organization) that was sent nationally and maybe beyond borders with the rationale that they wanted to collect a wide range of views on people with certain job characteristics. As there was no clear sample list of people with those characteristics, it is impossible to define the participation rate. It thus becomes impossible to define the prevalence of challenges faced by people with those job characteristics as generalizability is unknown. The survey may contribute to policy makers as it can have internal validity for comparisons and can raise issues. However, the prevalence estimates are not generalizable.

One can also argue about the trade-off of high participation within a narrowly defined homogeneous group versus low participation among a wider group. In our opinion, this is an artificial argument. One starts with narrowly defined homogeneous groups and works to expand knowledge about more widely defined groups. It is akin to the differences between clinical trials examining if substances can have intended positive effects versus pragmatic trials of what would happen if the substances were used in medical practice. They fundamentally answer different questions, and, as such, both are necessary.

Again, if there is no list of the target population, i.e. sampling frame, it is difficult to assess participation at recruitment. For example, the target population may be people with certain health problems like depression. In some countries, they can be recruited from general practitioners. Other countries have relied on volunteers recruited through advertisements [4]. With this latter approach and similar to our example of the online survey, the participation rate is totally unknown with corresponding unknown generalizability. However, the study can have internal validity. For target populations who are socially connected, respondent-driven sampling [7] can minimize bias. The sampling starts with a few individuals of the target population and then spreads through the social network. This sampling approach has been combined with online surveys by others [8] and by us.

Declining Participation in Research Studies

\section{What Can Be Done to Improve the Participation Rate?}

Across methods, regardless of mode of data collection, piloting is required as well as trust and outreach to the target population. The researcher should strongly consider using mixed modes of data collection to improve participation - such as online surveys followed by postal surveys - or other changes to the protocol. Repeated follow-up attempts regardless of method can increase the participation rate. In our experience, 3 mailings (baseline plus 2 follow-ups) in predefined intervals (usually 1-4 weeks apart) are an acceptable practice. As technology changes (e.g. online speed is becoming faster, more people use the Internet, landlines becoming more expensive), people need to examine when and where the studies were conducted when interpreting effectiveness/biases of different strategies.

One approach to enhancing participation is clearly articulated by Scott [9] and Scott et al. [10]. They designed and implemented an overall approach that is ethical and resulted in over $90 \%$ follow-up participation rates in hard-to-reach people, such as illegal drug users. The approach requires resources but it does not rely on just increasing incentives. It means hiring and training staff who are personable, flexible and receptive to ongoing training and monitoring. It also relies heavily on knowing the population and how people are potentially lost to follow-up and proactively arranging institutional agreements and obtaining consent at the time of recruitment to contact hospitals, jails, etc. The need for follow-up and the methods are explained to participants at recruitment and at every contact. Importantly, they did not wait until a follow-up assessment is due to begin to contact the person using comprehensive information collected at the time of recruitment on the individual and current/past social network. Finally the system relies on active monitoring to quickly make changes as needed.

Other recommendations for increasing the participation rate are specific for the mode of data collection and are presented in table 1. Advice on telephone surveys is based upon landline phones. With the mass movement to cell phones with their different use patterns and possibly different demographic characteristics, we need ongoing research to examine strategies and potential biases. Specific advice on postal and online surveys is based upon a recent Cochrane review [11]. For postal surveys, the Cochrane review found 110 different strategies tested. For online surveys, the Cochrane review found fewer trials and fewer strategies tested $(n=27)$.

Psychother Psychosom 2011;80:325-328 
Table 1. Recommendations for increasing participation rates by model of data collection

\begin{tabular}{ll}
\hline Mode & \\
\hline $\begin{array}{l}\text { Telephone } \\
\text { survey }\end{array}$ & $\begin{array}{l}\text { Do not use 'private' number; use an affiliation that } \\
\text { legitimizes the call } \\
\text { Interviewers need to properly introduce themselves } \\
\text { and the institution they represent }\end{array}$ \\
\hline Online & $\begin{array}{l}\text { Is the target sample online and technologically able } \\
\text { to complete the survey? } \\
\text { Nonmonetary incentives } \\
\text { Shorter questionnaires } \\
\text { Statement that others have responded } \\
\text { More interesting topic } \\
\text { Lottery } \\
\text { Offer of survey results } \\
\text { White screen background } \\
\text { Simple header } \\
\text { Deadline } \\
\text { Personalized questionnaire } \\
\begin{array}{l}\text { Picture included in the e-mail } \\
\text { Participation was reduced if the word 'survey' was } \\
\text { mentioned in the subject line, or the e-mail } \\
\text { included a male signature }\end{array} \\
\text { Importance of their participation } \\
\text { Trust of who is asking (i.e. university sponsorship, } \\
\text { prenotification, personalized questionnaires, } \\
\text { first-class stamped envelope with hand-written } \\
\text { address) } \\
\text { Lower barrier (i.e. monetary and nonmonetary } \\
\text { incentives, unconditional incentives, teaser on } \\
\text { envelope) } \\
\text { Follow-up to the initial mailing (i.e. contact, sending } \\
\text { new questionnaire) } \\
\text { gensitive nature }\end{array}$ \\
\hline Postal \\
surveys
\end{tabular}

\section{Conclusion}

Participation rates are definitely lower in the USA than they used to be. Lessons from other countries may inform ways to increase and maintain high participation rates. This decline means we as readers must be suspicious, especially of findings with small effect sizes, and examine the recruitment process and retention for potential biases. If the authors do not present sensitivity analyses, then we need to be analytically agile enough to do it. The decline means that we as researchers must be transparent about the recruitment process, devote resources to maximizing it and probe for biases by additional analyses. Importantly, statistical techniques will not solve the problem. It means that we as reviewers must demand the participation rate be honestly and prominently presented. It does not mean that we reward studies with high participation but meager contributions and discard innovative studies on the basis of an arbitrary participation rate number. It means we must continue to be intelligent and critical consumers of research findings. If the findings are robust across studies with different biases, then it is more likely that the findings can be generalized. Finally, although it is not often stated, it means that we as funders are paying more as the lower participation rates mean more effort has to be expended to obtain the target sample size.

\section{References}

1 Galea S, Tracy M: Participation rates in epidemiologic studies. Ann Epidemiol 2007;17: 643-653.

2 Agoritsas TA, Deom M, Perneger TV: Study design attributes influenced patients' willingness to participate in clinical research: a randomized vignette-based study. J Clin Epidemiol 2011;64:107-115.

3 Lee S, Brown E, Grant D, Belin T, Brick J: Exploring nonresponse bias in a health survey using neighborhood characteristics. Am J Public Health 2009;99:1811-1817.

4 Brauzer B, Goldstein BJ: Symptomatic volunteers: another patient dimension for clinical trials. J Clin Pharmacol 1973;13:89-98.
5 Taylor M: What is sensitivity analysis? Hayward Medical Communications. http:// www.medicine.ox.ac.uk/bandolier/:/ painres/download/whatis/What_is_sens_ analy.pdf (accessed January 13, 2011).

6 Solomon D: Conducting web-based surveys. Pract Assess Res Evaluat 2001, vol 7, No 19.

7 Heckathorn D: Respondent-driven sampling: a new approach to the study of hidden populations. Social Problems 1997;44:174-199.

$\checkmark 8$ Wejnert C, Heckathorn D: Web-based network sampling: efficiency and efficacy of respondent-driven sampling for online research. Sociol Methods Res 2008;37:105134.
-9 Scott C: A replicable model for achieving over $90 \%$ follow-up rates in longitudinal studies of substance abusers. Drug Alcohol Depend 2004;74:21-36.

10 Scott C, Sonis J, Creamer M, Dennis M: Maximizing follow-up in longitudinal studies of traumatized populations. J Traumat Stress 2006;19:757-769.

11 Edwards PJ, Roberts I, Clarke MJ, Diguiseppi C, Wentz R, Kwan I, Cooper R, Felix LM, Pratap S: Methods to increase response to postal and electronic questionnaires. Cochrane Database Syst Rev 2009;3:MR000008. 